\title{
EFECTO DEL MANEJO DE LOS RESIDUOS DE EXPLOTACION DE PINUS RADIATA EN LOS PROCESOS DE EROSION
}

Cesar Alarcón A. José Antonio Prado(1)

\section{RESUMEN}

En este estudio se cvalúa el efecto de distintas modalidades de manejo de los residuos de explotación de un bosque de Pinus radiata en los procesos erosivos que se inician con la explotación. Estas modalidades incluyen la quema empleando distintos sistemas de encendido; la cosecha intensiva de la biomasa y la permanencia del desecho sin mayor alteración

El método empleado solo permite una estimación de la erosión producida, la que de acuerdo a los resultados, podría llegar hasta los $160 \mathrm{~m}^{3}$ de suelo por ha.

Desde el punto de vista nutricional, las pérdidas no son alarmantes, pero tampoco despreciables.

\begin{abstract}
This study evaluates the effect of different alternatives of Pinus radiata logging slash disposal on the erosion processes produced after the harvest.

These alternatives include slashbuming under different conditions; intensive harvest; and undisturbed logging slash.

The methods used to evaluate erosion just allowan estimate of the process, which in this case would be removing as much as $160 \mathrm{~m}^{3} / \mathrm{ha}$ of soil, in the most affected area.

From the nutritional point of view, the losses are not alarming, but considerable.
\end{abstract}

(1) Ing. Forestales, Instituto Forestal - Huérfanos 554, Santiago - Chile 


\section{INTRODUCCION}

El tratamiento de los desechos de explotación es un tema que preocupa a forestadores e investigadores, ya que tiene una importante incidencia en los costos de reforestación y en la productividad del sitio, especialmente en el largo plazo.

La extracción total de la biomasa puede incrementar notablemente el monto de materia prima y combustible producidos por el bosque, pero al mismo tiempo produce un considerable aumento en la exportación de nutrientes desde el sitio, lo cual, tarde o temprano, dependiendo de la calidad de éste, afectará su productividad.

La extracción total no es una práctica común en Chile; todavía se deja una cantidad considerable de biomasa como desecho de la explotación. Este material, en la mayoría de los casos, es apilado y quemado, con lo cual se facilitan las faena de replante, se disminuye el peligro de incendios y se produce una considerable liberación de nutrientes, que quedan a disposición de las plantas recién establecidas, provocando un desarrollo inicial vigoroso.

Estas ventajas tienen su costo. El fuego consume gran cantidad de materia orgánica, lo cual se traduce en una importante pérdida de nutrientes por volatilización y por el posterior arrastre de las cenizas por la acción del viento y principalmente del agua, que los puede llevar hacia los caudales o hacia horizontes profundos en el suelo, en donde no son alcanzados por los árboles. El fuego también puede tener un efecto negativo en las propiedades físicas del suelo. Este al quedar descubierto, puede verse afectado por procesos crosivos que arrastran, junto a sus partículas una considerable cantidad de nutrientes. Este efecto negativo es variable y dependerá de las condiciones del suelo y del tratamiento que se de a los desechos.

La erosión en los ecosistemas comienza cuando la acción antropógena altera el equilibrio natural del suelo, sin pensar en sus limitaciones, quedando expuesto a la acción directa de la lluvia y el arrastre posterior por las aguas de escurrimiento superficial en la pendiente. Esta acción genera una perdida a veces imperceptible y permanente de las capas más finas y superficiales del suelo, como coloides orgánicos y minerales, materia orgánica (humus) y arcilla, todos elementos bases del intercambio de nutrientes entre el suelo y las plantas.

La susceptibilidad a la erosión de un suelo es función de un número de factores: la intensidad de la lluvia, su capacidad de infiltración, la pendiente, las propiedades químicas y físicas propias que controlan la desintegración de las partículas y determinan la cohesión del suelo, y la vegetación existente. Estos últimos factores son los que determinan la estabilidad y la capacidad de infiltración.

Una de las causas más importantes en la pérdida de nutrientes es la erosión provocada por la remoción de los árboles en la explotación. El madereo, la construcción de caminos, la utilización de maquinaria pesada, el tratamiento de los residuos mediante quemas controladas, pueden generar de escasos a fuertes procesos erosivos y obviamente, la pérdida de los nutrientes contenidos en el suelo. Este fenómeno afecta considerablemente la productividad del sitio (Mac Coll and Grigal, 1979, Patric, 1978; Stone, 1973).

El Instituto Forestal, en cooperación con las mayores empresas del sector, ha instalado cuatro ensayos que tienen como objetivo el determinar el efecto del tratamiento de los 
desechos en la productividad de la segunda rotación de Pinus radiata. En este caso an alizan los efectos de la presencia de los desechos y de su eliminación mediante una cosecha intensiva o el uso del fuego, en los procesos erosivos.

\section{MATERIAL Y METODO}

\section{Tratamientos}

Con el fin de establecer el efecto de distintos métodos de tratamiento de los desechos de explotación en la productividad del sitio se han establecido cuatro ensayos, que consideran los siguientes tratamientos.

T1: Explotación tradicional con apilado del desecho y quema controlada. Técnica de encendido: fuego en retroceso.

T2: Explotación tradicional, desecho sin apilar y quema controlada. Técnicas de encendido: fucgo en retroceso.

T3: Explotación tradicional, desecho sin apilar, quema controladas. Técnica de encendido: fuego frontal.

T4: Explotación tradicional, desecho sin apilar, quema controlada. Técnica de encendido: optativa (fuego circular, por focos, por flancos o por fajas), de acuerdo a las condiciones de cada lugar.

T5: Explotación tradicional, sin apilado y sin quema de residuos.

T6: Explotación intensiva, sin quema de residuos y retiro de todo material de diámetro supcrior a $3 \mathrm{~cm}$.

En la explotación tradicional, se efectuó el madereo con tractor articulado y se aprovechó cl fuste hasta un diámetro aproximado a $5 \mathrm{~cm}$. En la explotación intensiva, también se realiza el madereo con tractor articulado, pero se retiró todo el material de fuste y ramas, hasta un diámetro de $3 \mathrm{~cm}$. El resto del material queda esparcido, sin quemar.

La superficie total que abarcó cada tratamiento, fue de 5 a 19 hectáreas de extensión para los tratamientos 2 al $6 \mathrm{y} \mathrm{de} 900 \mathrm{~m}^{2}$ para el tratamiento 1 .

\section{Lugares de Ensayo}

Para realizar esta investigación se cligieron cuatro lugares, representativos de los suclos en que se encuentra la mayoría de las plantaciones de Pinus radiata.

La ubicación y algunos antecedentes básicos de cada lugar se entregan en el Cuadro 1. 


\section{CUADRO 1}

\section{UBICACION Y ANTECEDENTES BASICOS DE SUELO Y CLIMA DE LOS LUGARES DE ENSAYO}

\begin{tabular}{|c|c|c|c|c|c|c|c|}
\hline \multirow[t]{2}{*}{ Predio } & \multirow[t]{2}{*}{ Región } & \multicolumn{3}{|c|}{ Suelo } & \multicolumn{2}{|c|}{ Clima } & \multirow[t]{2}{*}{ Topografía } \\
\hline & & Mat. Origen & Serie & Profundidad & PPT (mm) & $\begin{array}{l}T^{0} \\
\text { Media }\end{array}$ & \\
\hline San Pedro & VII & Metamórfico & Constitución & Profundo $(1.5 \mathrm{~m})$ & $>1.000$ & 13.9 & Ondulada \\
\hline Maquehua & VIII & Arenas Volcánicas & Coreo & Profundo $(2.0 \mathrm{~m})$ & $>1.200$ & 13.8 & Plana \\
\hline Meñir & VIII & Granítico & Cauquenes & Profundo $(1.2 \mathrm{~m})$ & $>1.200$ & 13.6 & Ondulada \\
\hline La Colcha & VIII & Metamórfico & Colico & Profundo $(1.0 \mathrm{~m})$ & $>1.600$ & 13.3 & Ondulada \\
\hline
\end{tabular}

\section{Sistema de Medición}

Las mediciones exactas de pérdida de suelo por erosión hídrica, se pueden obtener para un área determinada, mediante la colección de sedimentos de una parcela aislada, en receptáculos o colectores; midiéndose los kilos de suelo que se pierden en un terreno con pendiente y cobertura vegetal determinada. Su uso es costoso y requiere varios años de medición para cuantificar el fenómeno erosivo.

En este caso se ha empleado un método alternativo, sin captura de sedimento, propuesto por Dunne (1977).

El método consiste en la instalación de parcelas de $25 \mathrm{~m}^{2}$, sobre las cuales se realiza un reticulado y se instala un clavo por cada $\mathrm{m}^{2}$.

Se emplean clavos de 5", que junto a una golilla delgada de 1" de diámetro, se entierran hasta el nivel del suelo. Posteriormente se mide el descenso de la golilla y nuevamente se entierra el clavo hasta el nivel del suclo. Esto último tiene por objeto minimizar el mayor problema que ha encontrado el empleo de este sistema, que es el hurto de los clavos; al enterrarlos nuevamente, quedan menos visibles y hay más posibilidades de que permanezcan en su sitio.

En cada uno de los cuatro predios se instalaron 24 parcelas, 4 por cada uno de los tratamientos generales, lo que da un total de 96 parcelas de medición.

Hasta la fecha se han realizado dos mediciones, las que debido al problema señalado con anterioridad, se encuentran incompletas. El mayor problema se presentó en la primera medición, ya que en algunos predios sólo se encontró un escaso porcentaje de los clavos,lo que obligó a reinstalar los ensayos, y a eliminar de este análisis el predio Meñir. Los datos de la Colcha, están basados en parcelas incompletas.

\section{Variables de Análisis}

Con el empleo del sistema de medición se registran dos tipos de movimientos de suelo 
dentro de cada parcela: un movimiento de arrastre, que deja el clavo descubierto y un movimiento de agregación, por la erosión que se produce más arriba en la pendiente. Ambos movimientos se registran con signos negativo y positivo, respectivamente.

La pérdida efectiva de suelo (PE) será la diferencia entre arrastre y agregación. La otra variable que se emplea es el movimiento total de arrastre (MTA). En la realidad, debido al sistema de parcelas abiertas, esta variable está subestimada, ya que el total de las mediciones negativas representa solo parte del volumen total de suelo en movimiento.

\section{Análisis de Suelo}

Como parte de la investigación, se tomaron muestras de suelo a distinta profundidad. El contenido de nutrientes y la densidad aparente en la muestra superficial se emplea para estimar la pérdida de nutrientes producidos por la erosión.

\section{Diseño y análisis estadístico}

El diseño empleado es el de parcelas al azar, con 4 repeticiones.

La comparación de los tratamientos se realiza mediante análisis de varianza y el test de comparaciones múltiples de Tukey.

\section{RESULTADOS}

En el Cuadro 2 se entregan los resultados, expresados en $\mathrm{m}^{3} / \mathrm{ha}$, de los dos tipos de movimiento de suelo que se han considerado como variables para este análisis.

Para cada uno de los controles y para cada predio se entrega la pérdida efectiva (PE) y el movimiento total de arrastre (MTA).

Después del primer año, al analizar las variables PE y MTA se visualiza una tendencia al aumento desde el tratamiento 1 hacia el tratamiento 6, con un máximo en el tratamiento 5, en dos de los lugares de ensayo (Maquehua y La Colcha). Este hecho resulta sorprendente ya que se supone que este tratamiento, en el cual no se quemaron ni retiraron los desechos, debería presentar la menor tasa de erosión.

El tratamiento 6, también en contra de lo esperado, ocupa el segundo lugar en cuanto a pérdida de suelo en Maquehua y La Colcha, y es el que presenta la mayor tasa de erosión en San Pedro. En este caso en particular, la cosecha fue realmente intensiva, ya que se sacó prácticamente toda la biomasa (no se dejó el material con diámetros menores a $3 \mathrm{~cm}$ ) dejando el suelo completamente expuesto. A esto se suma el hecho de que estas parcelas tienen una mayor pendiente.

La mayor erosión detectada en los tratamientos 5 y 6 tendría su explicación en el movimiento superficial de la capa de acículas en distintos grados de descomposición, la cual, en los otros casos desapareció con la quema. Aparentemente, cualquiera sea el tratamiento, se produce una importante pérdida de materia orgánica en descomposición. 


\section{CUADRO 2}

\section{PERDIDA EFECTIVA Y MOVIMIENTO TOTAL DE SUELO SEGUN LUGAR DE ENSAYO \\ (metros cúbicos por hectáreas)}

\begin{tabular}{|l|c|r|r|r|r|r|r|}
\hline \multirow{2}{*}{ Predio } & & \multicolumn{2}{|c|}{ CONTROL 1 } & \multicolumn{2}{c|}{ CONTROL 2 } & \multicolumn{2}{c|}{ CONTROL 1 + 2 } \\
\cline { 3 - 8 } & Trat. & $\begin{array}{c}\text { Pérd. } \\
\text { Efec. }\end{array}$ & $\begin{array}{c}\text { Mov. } \\
\text { Tot. }\end{array}$ & $\begin{array}{c}\text { Pérd. } \\
\text { Efect. }\end{array}$ & $\begin{array}{c}\text { Mov. } \\
\text { Tot. }\end{array}$ & $\begin{array}{r}\text { Pérd. } \\
\text { Efect. }\end{array}$ & $\begin{array}{c}\text { Mov. } \\
\text { Tot. }\end{array}$ \\
\hline San Pedro & 1 & 8,64 & 42,19 & 30,16 & 56,28 & 38,80 & 98,47 \\
& 2 & 6,82 & 52,27 & 58,37 & 76,40 & 65,19 & 128,68 \\
& 3 & 25,56 & 76,65 & 31,18 & 58,24 & 56,74 & 134,88 \\
& 4 & 31,89 & 104,43 & 45,04 & 83,55 & 76,93 & 187,98 \\
& 5 & 37,94 & 84,73 & 31,20 & 80,52 & 69,14 & 165,25 \\
& 6 & 58,41 & 111,44 & 39,56 & 70,88 & 97,97 & 182,32 \\
\hline Maquehua & 1 & 2,00 & 47,96 & 11,73 & 59,31 & 13,73 & 107,27 \\
& 2 & 23,29 & 65,16 & 14,72 & 53,58 & 38,01 & 118,74 \\
& 3 & 14,89 & 49,87 & 27,78 & 84,12 & 42,67 & 133,99 \\
& 4 & 9,61 & 37,48 & 23,38 & 63,25 & 32,99 & 100,73 \\
& 5 & 98,00 & 157,24 & 62,48 & 97,99 & 160,48 & 255,22 \\
& 6 & 46,69 & 104,04 & 37,66 & 85,15 & 84,35 & 189,19 \\
& 1 & 0,59 & 43,37 & 14,11 & 52,01 & 14,70 & 95,38 \\
& 2 & 16,93 & 59,65 & 30,07 & 65,31 & 47,00 & 124,95 \\
& 3 & 34,95 & 73,00 & 2,29 & 37,78 & 37,24 & 110,78 \\
& 4 & 3,92 & 73,82 & 25,53 & 61,65 & 29,45 & 135,47 \\
& 5 & 21,91 & 100,26 & 81,01 & 93,90 & 102,92 & 194,16 \\
& 6 & 28,47 & 76,68 & 58,09 & 74,34 & 86,56 & 151,02 \\
\hline
\end{tabular}

Después del primer año, el tratamiento 1, que es la explotación tradicional con apilado del desecho, sería el más consistente en cuanto a una menor tasa de erosión, con valores entre 42 y $48 \mathrm{~m}^{3}$ /ha, para la variable "movimiento total de arrastre" (MTA). Este tratamiento también presenta los menores valores de pérdida efectiva(PE), por lo que en todos los lugares casi se compensó la extracción con el movimiento de aporte.

Durante el segundo año se aprecia una disminución en el movimiento total de arrastre. En San Pedro esta alcanza a un 9,7\% para el promedio de todos los tratamientos, en tanto que en Maquehua y La Colcha las reducciones son de $4 \%$ y $9,8 \%$ respectivamente. La pérdida efectiva, en cambio, experimenta aumentos de $39.1 \%$ en San Pedro, y de un 97.7\% en La Colcha cuando se analizan todos los tratamientos en conjunto. En Maquehua se produce una ligera disminución.

Los tratamientos 1 y 3 presentan, en este caso, la tendencia a una menor tasa de erosión.

El aumento en la pérdida efectiva puede ser un hecho fortuito, al producirse menos 


\section{GRAFICO 1}

MOVIMIENTO TOTAL Y PERDIDA EFECTIVA DE SUELO EN PREDIOS DE ENSAYOS
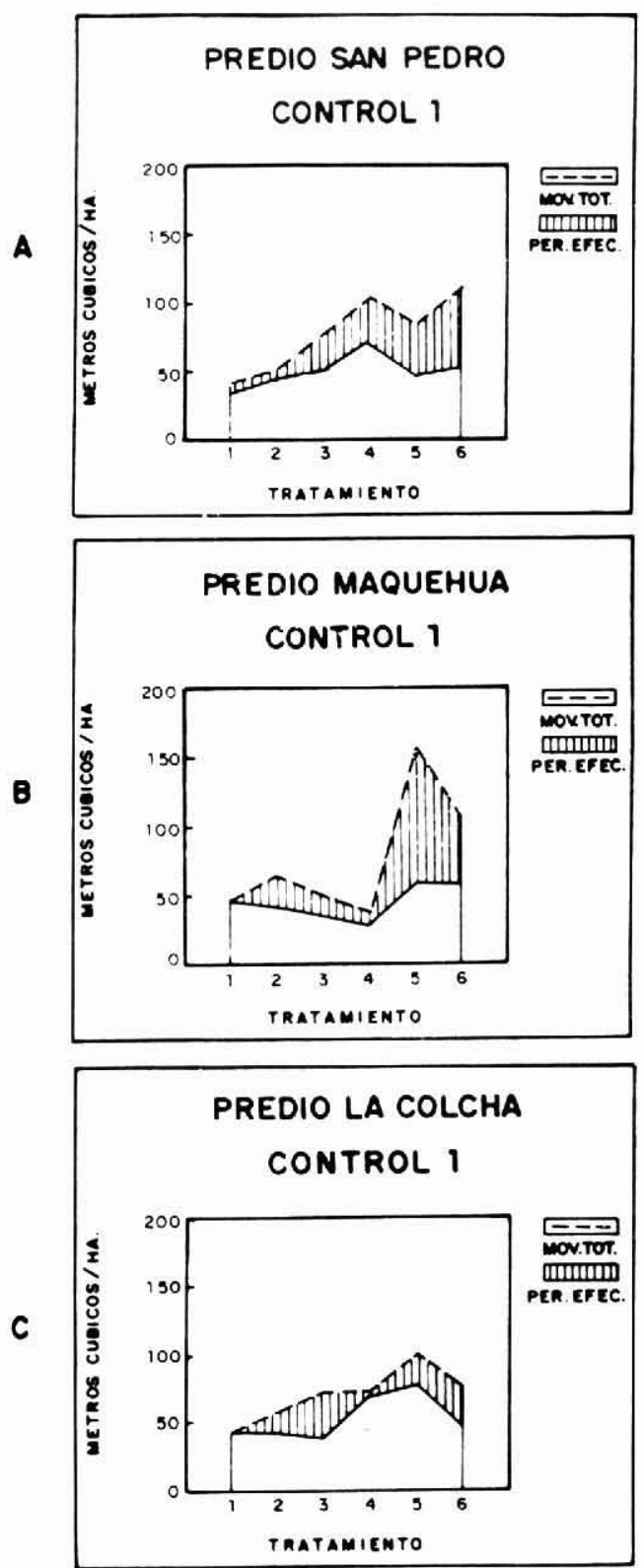
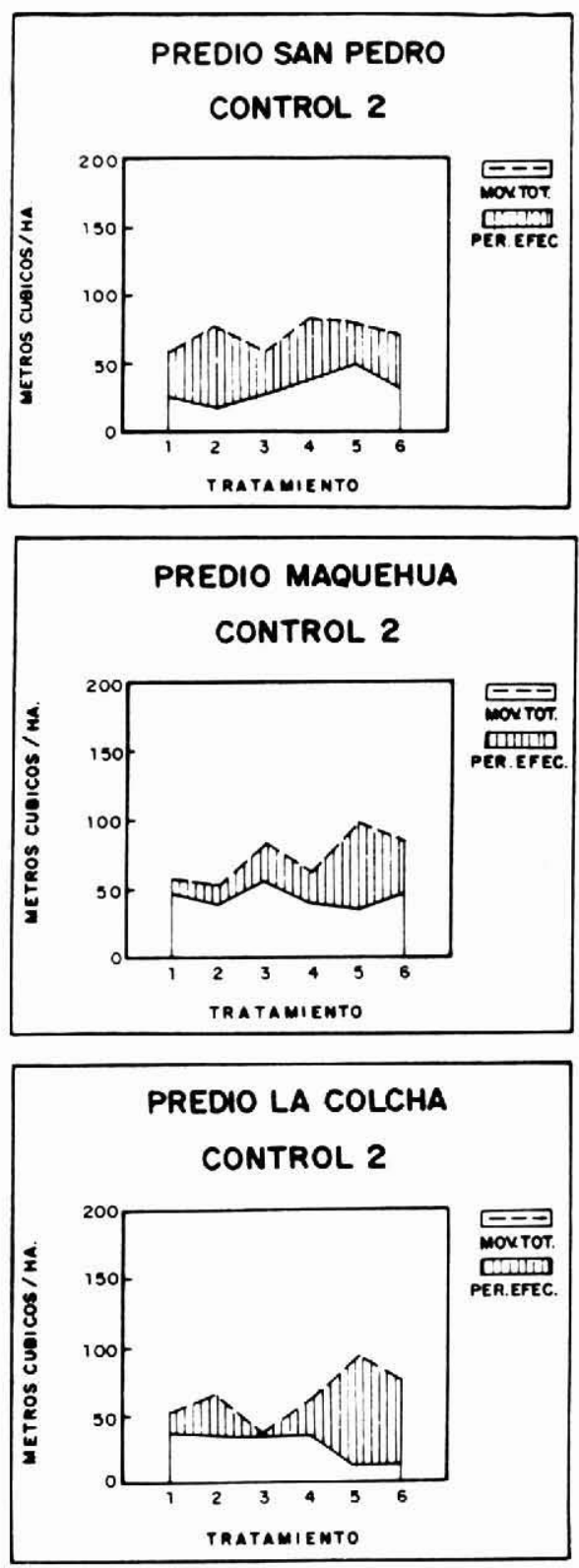
depósito en las parcelas de medición que en el año anterior, generándose una mayor diferencia, aún cuando el movimiento total de arrastre fuese inferior.

La disminución en el movimiento total durante el segundo año puede estar relacionada a una mayor estabilidad en el suelo o al hecho de que 1988 fue considerablemente menos lluvioso que el año anterior. En San Pedro, sólo en Julio de 1987 cayeron 806 mm.

Las pérdidas acumuladas durante los dos años (Gráficos 2a a 2c) son considerables en todos los lugares de ensayo. Llama la atención el nivel de pérdida de suelo al que se llega en Maquehua, a pesar de la escasa pendiente del área del ensayo.

$\mathrm{Al}$ analizar estadísticamente los resultados encontramos que solo en La Colcha existen diferencias significativas a un $95 \%$ de confianza cuando se compara la pérdida efectiva en el segundo control. (Cuadro 3).

\section{CUADRO 3}

\section{RESULTADO DEL ANALISIS DE VARIANZA PARA CADA PREDIO}

\begin{tabular}{|l|c|c|c|c|}
\hline \multirow{2}{*}{ Predio } & \multicolumn{2}{|c}{ CONTROL 1 } & \multicolumn{2}{c|}{ CONTROL 2 } \\
\cline { 2 - 5 } & \multicolumn{2}{|c|}{ (Noviembre 1987) } & \multicolumn{2}{c|}{ (Noviembre 1988) } \\
\cline { 2 - 5 } & Per. Efec. & Mov. Tot. & Per. Efec. & Mov. Tot. \\
\hline \multirow{3}{*}{$\begin{array}{l}\text { San Pedro } \\
\text { Maquehua }\end{array}$} & NS & NS & NS & NS \\
La Colcha & NS & NS. & NS & NS \\
& & NS. & $*$ & NS. \\
\hline
\end{tabular}

NS: No existen diferencias significativas entre los tratamientos.

*: Si existen diferencias significativas al nivel de $\alpha=0.05$

.: Modelo no aditivo.

Al realizar el test de comparación múltiple de Tukey se encuentra que la diferencia estadísticamente significativa se produce al comparar los tratamientos 3 y 5 (Cuadro 4). 
GRAFICO 2

MOVIMIENTO TOTAL Y PERDIDA EFECTIVA ACUMULADA DE LOS DOS CONTROLES EN PREDIOS DE LOS ENSAYOS

A

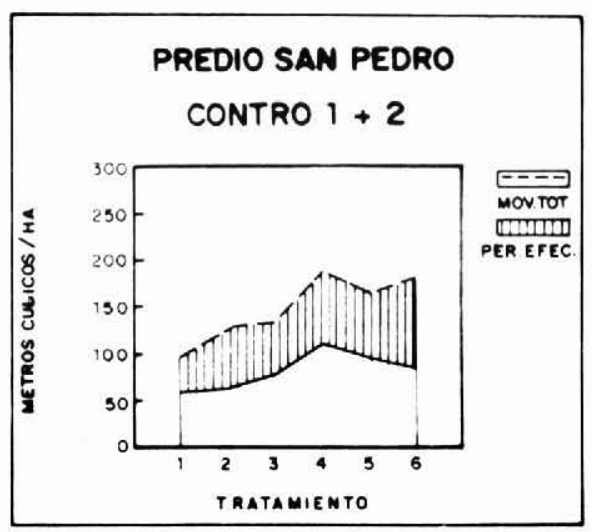

B

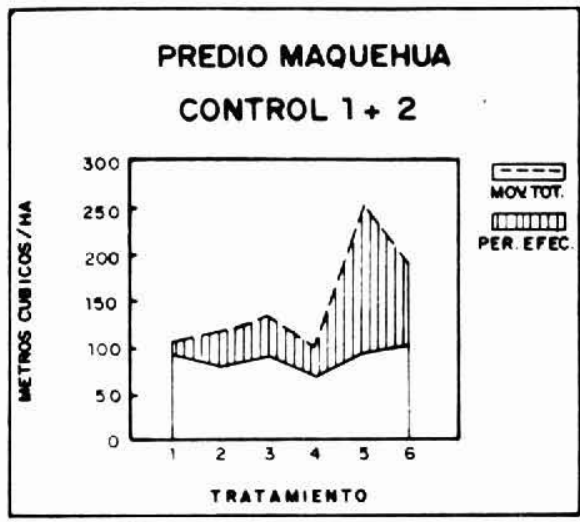

C

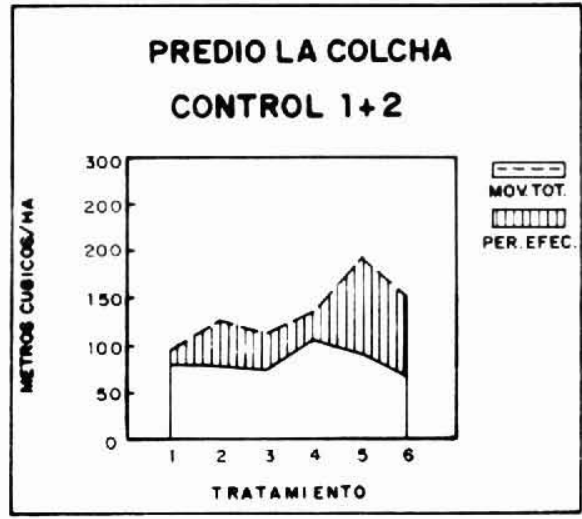

Volumen 4, Número 1, 1990 / 43 


\section{CUADRO 4}

\section{RESULTADO DEL TEST DE COMPARACION MULTIPLE DE LOS TRATAMIENTOS EN LA COLCHA}

\begin{tabular}{|c|c|c|c|c|c|c|}
\hline & \multicolumn{6}{|c|}{ TRATAMIENTOS } \\
\hline & 5 & 6 & 2 & 4 & 1 & 3 \\
\hline $\begin{array}{l}\text { Pérdida Efectiva } \\
\left(\mathrm{m}^{3} / \mathrm{ha}\right)\end{array}$ & 81.01 & 58.09 & 30.07 & 25.53 & 14.12 & 2.29 \\
\hline
\end{tabular}

Las líneas indican tratamientos sin diferencias significativas $\alpha=0.05$.

\section{DISCUSION}

El hecho de que las parcelas no hayan sido aisladas, impide estimar el volumen efectivo de suelo que se pone en movimiento producto de la erosión. Este dato se habría logrado al aislar cada una de las parcelas, impidiendo el aporte desde el exterior.

De acuerdo a los resultados de este ensayo queda en evidencia que no todo el material que inicia su movimiento en la ladera es transportado fuera del "sistema", por lo que al establecer parcelas de medición cerradas, sin aporte, se estaría haciendo una sobreestimación del material efectivamente removido fuera del área afectada por la erosión.

El sistema empleado en este estudio puede dar, después de varios años de observación, hasta que el proceso erosivo se estabilice, una aproximación bastante cercana de la pérdida real del suelo. Sin embargo, la experiencia obtenida en este estudio indica que es necesario hacer un establecimiento más detallado del ensayo, que permita reconstituir la parcela, aún cuando se pierdan los clavos. Esto puede lograrse extendiendo una cuerda (entre dos estacas permanentes), sobre las líneas de clavos y midiendo la distancia desde esta rasante hasta la cabeza del clavo. En cada medición se extendería la cuerda entre las estacas y se mediría la distancia hasta el suelo. El clavo sólo cumpliría la función de señalar el punto donde debe realizarse la medición. Si el clavo falta es fácil de restablecer el lugar de medición, por distancia sobre la línea desde las estacas o desde algún clavo adyacente.

Otro factor importante que debe ser considerado es la pendiente de las parcelas de medición. En este caso hay bastante variabilidad.

A pesar de estas limitaciones se ha podido estimar, al cabo de 2 años, la pérdida efectiva de suelo, especialmente en los tratamientos con quema. En los tratamientos 5 y 6 es probable que la pérdida de suelo mineral este sobrestimada por una medición de las pérdidas producidas en el mantillo en descomposición. Esta sería la manera de explicar el por qué en 
estos tratamientos, en que el suelo ha quedado con cierta protección, se produce una pérdida mayor.

Hasta el segundo año después del establecimiento de los ensayos, se ha estimado que las pérdidas efectivas totales fluctúan entre los 38,8 y $98 \mathrm{~m}^{3} / \mathrm{ha}$ en San Pedro; 13,7 y $160,5 \mathrm{~m}^{3} / \mathrm{ha}$ en Maquehua y 14,7 y $102,9 \mathrm{~m}^{3} /$ ha en La Colcha.

Esta pérdida de suelo es irreparable, ya que la longitud de la rotación no es suficiente para permitir su recuperación. Lo que si es posible es reponer parte de los elementos que han sido arrastrados por los procesos erosivos.

Si se asume que la erosión se ha producido en los 5 primeros $\mathrm{cm}$ del suelo y como se determinó su contenido de nutrientes, densidad aparente, se puede estimar esta pérdida.

En el Cuadro 4 aparece la pérdida producida durante los dos primeros años, en base a los datos de pérdida efectiva producida en cada parcela.

\section{CUADRO 4}

PERDIDA DE NUTRIENTES (NPK) PRODUCIDA POR EFECTO DE LA EROSION HASTA EL SEGUNDO AÑO DESPUES DE LA EXPLOTACION

\begin{tabular}{|l|c|c|c|c|}
\hline \multirow{2}{*}{ Predio } & \multirow{3}{*}{ Tratamiento } & \multicolumn{3}{|c|}{ Pérdida (Kg/ha) } \\
\cline { 3 - 5 } & & N & P & K \\
\cline { 3 - 5 } & & 0,88 & 0,32 & 9,66 \\
\multirow{2}{*}{ San Pedro } & T1 & 2,17 & 0,56 & 21,39 \\
& T2 & 1,65 & 0,47 & 11,83 \\
& T3 & 3,09 & 0,31 & 16,48 \\
& T4 & 1,12 & 0,38 & 10,53 \\
& T5 & 4,10 & 0,40 & 18,50 \\
& T6 & 0,42 & 0,77 & 1,38 \\
\multirow{2}{*}{ Maquehua } & T1 & 1,01 & 3,05 & 3,84 \\
& T2 & 1,50 & 3,17 & 4,36 \\
& T3 & 0,95 & 2,60 & 4,01 \\
& T4 & 7,49 & 10,58 & 21,36 \\
& T5 & 3,80 & 3,90 & 10,27 \\
& T6 & 0,93 & 0,06 & 3,30 \\
& T1 & 2,41 & 0,14 & 11,73 \\
& T2 & 0,30 & 0,02 & 1,30 \\
& T3 & 1,64 & 0,09 & 5,43 \\
& T4 & 4,42 & 0,61 & 22,06 \\
& T5 & 4,12 & 0,41 & 34,07 \\
\hline
\end{tabular}


Las pérdidas efectivas producidas en los dos primeros años no son alarmantes, pero tampoco despreciables. Las mayores pérdidas se producen en los tratamientos 5 y 6 , pero como ya se mencionó, puede haber cierta distorsión en cuanto a la pérdida real de suelo mineral en estos tratamientos, ya que es probable que se haya incluido en la medición parte del humus. Este arrastre de materia orgánica, que no ha sido registrado, implica una considerable pérdida de nutrientes.

Para mantener la productividad del sitio, será necesario reponer, tarde o temprano, dependiendo del nivel de fertilidad natural, las disponibilidades y reservas existentes en el suelo, los nutrientes perdidos por la erosión, mediante la aplicación de fertilizante.

Bengston (1981), señala que el aprovechamiento del fertilizante es, por lo general, muy poco eficiente. Por lo tanto para que el sistema alcance el mismo nivel de nutrientes que existía antes de la explotación, es necesario aplicar fertilizantes en una tasa tal que doble el monto de nutrientes extraidos. Aún cuando esta no es una regla general, ya que depende de las condiciones del suelo y otros factores relacionados al tipo de fertilizante, vegetación existente y forma de aplicación, se ha decidido emplear este criterio para destacar la importancia que eventualmente pueden tener estas pérdidas. Buscando la combinación óptima de fertilizantes, desde el punto de vista del costo, se calcularon los valores que sc entregan en el Cuadro 5.

\section{CUADRO 5}

\section{COSTO DE REPOSICION DE LAS PERDIDAS REALES Y POTENCIALES DE NUTRIENTES DEBIDO A LA EROSION}

\begin{tabular}{|l|c|c|c|}
\hline \multirow{2}{*}{ Predio } & \multirow{2}{*}{ Tratamiento } & P. Real & P. Potencial \\
\cline { 3 - 4 } & & \$/ha & \$/ha \\
\hline \multirow{2}{*}{ San Pedro } & T1 & 3835 & 9929 \\
& T2 & 8526 & 16338 \\
& T3 & 4911 & 11662 \\
& T4 & 6974 & 16898 \\
& T5 & 4245 & 10305 \\
& T6 & 8046 & 15012 \\
\hline \multirow{2}{*}{ Maquehua } & T1 & 900 & 7358 \\
& T2 & 2778 & 8589 \\
& T3 & 3163 & 9973 \\
& T4 & 2655 & 8437 \\
& T5 & 13774 & 21936 \\
& T6 & 6261 & 14043 \\
\hline \multirow{2}{*}{ La Colcha } & T1 & 1496 & 12090 \\
& T2 & 5001 & 14075 \\
& T3 & 565 & 8648 \\
& T4 & 2491 & 12277 \\
& T5 & 9497 & 22303 \\
\hline
\end{tabular}


$\mathrm{Al}$ analizar estas cifras hay que considerar que esta es una pérdida parcial, que pucde incrementarse considerablemente, ya que el volumen de suelo en movimiento ladera abajo es por lo menos igual al volumen entregado por las mediciones negativas, que se ha denominado "movimiento total". El costo de reposición de los nutrientes que eventualmente podrían salir del sistema también se entregan en el Cuadro 5.

Estas cifras en ningún caso bajan de los $\$ 8.000 /$ ha (US\$32/ha), llegando a más de $\$$ 22.000 , en La Colcha. Las pérdidas potenciales por efecto de la erosión son considerables.

Es importante recordar que la erosión es sólo uno de los factores que afecta negativamente la productividad del sitio. Kunz et al. (1985) señalan que en la explotación solo en el fuste y la corteza se extraen $140 \mathrm{~kg} / \mathrm{ha}$ de N, $20,4 \mathrm{~kg} / \mathrm{ha}$ de $\mathrm{P}$ y $183,9 \mathrm{~kg} / \mathrm{ha}$ de $\mathrm{K}$. Si se agregan las pérdidas producidas por la quema, estas cifras suben a $384 \mathrm{~kg} / \mathrm{ha}$ de N; $34,8 \mathrm{~kg} / \mathrm{ha}$ de $\mathrm{P}$ y 248 $\mathrm{kg} / \mathrm{ha} \mathrm{de} \mathrm{K}$. La "exportación de estos dos últimos elementos sube considerablemente (a 59 y $396 \mathrm{~kg} / \mathrm{ha}$ respectivamente) si se realiza una explotación intensiva, en que gran parte de la biomasa es extraida (tratamiento 6).

La reposición de estos tres elementos costaría, en el caso de una explotación tradicional, con quema, $\$ 121.000 /$ ha y $\$ 227.000$ en el caso de la explotación intensiva, a lo cual habría que agregar las pérdidas producidas por la erosión que se han calculado en este trabajo.

\section{CONCLUSIONES}

Debido a la forma en que han sido establecidos los ensayos, resulta difícil obtener conclusiones claras en cuanto el efecto de los distintos tratamientos en los proceso erosivos, cn lo que va transcurrido desde la instalación del ensayo. Esto se debe, principalmente, a que existe una considerable cantidad de material en movimiento, que aún no ha salido del sistema. La pérdida real debido a la erosión, sólo podrá evaluarse una vez que el proceso se haya detenido.

Los datos obtenidos hasta el momento permiten visualizar que el problema de la crosión es bastante grave, poniendo en movimiento importantes volúmenes de suelo en todos los lugares de ensayo.

De acuerdo a las estimaciones realizadas las pérdidas totales de suelo fluctúan entre los 13.7 y $160.5 \mathrm{~m}^{3} / \mathrm{ha}$ en tanto que el material en movimiento varia entre los 95.4 y $255 \mathrm{~m}^{3}$ / ha.

Las pérdidas de nutrientes por efecto de la erosión varían de 0.4 a $7.5 \mathrm{~kg} / \mathrm{ha}$, en el caso del Nitrógeno; de 0.02 a $10.6 \mathrm{~kg} / \mathrm{ha}$ de P y de 1.3 a $34.1 \mathrm{~kg} / \mathrm{ha}$ de $\mathrm{K}$.

Las distintas modalidades empleadas en los tratamientos con quema hasta el momento no presentan diferencias estadísticamente significativas. Al analizar la pérdida efectiva y el movimicnto total acumulados durante los dos años, se ve que el tratamiento tradicional es el que registra los menores valores, en los tres lugares de ensayo, lo cual no concuerda con lo que cra lógico esperar esto es, que los tratamientos sin quema, especialmente el tratamiento 5, que deja los desechos en el terreno, fuesen los menos afectados por la erosión.

Es también inconsistente el hecho de que los tratamientos 5 y 6 registren altos valores de pérdida y movimiento total, lo cual se debe a una sobreestimación, producto de la instalación de las gotillas sobre acículas o al menos, sobre materia orgánica en descomposición. 


\section{RECONOCIMIENTOS}

El presente trabajo forma parte del proyecto "Efecto de los diferentes métodos d utilización y manejo de los residuos de explotación en el rendimiento de la segunda rotaciór de plantaciones de Pino Insigne", que realiza el Instituto Forestal por encargo de la Corporaciór de Fomento de la Producción, CORFO, con el apoyo técnico y financiero de las siguiente: empresas: Bosques de Chile, Forestal Arauco, Forestal Cholguán, Forestal Mininco, Foresta Río Vergara y Forestal Tornagaleones.

\section{REFERENCIAS BIBLIOGRAFICAS}

1. BENGSTON, G.W. 1981. Nutrient conservation in forestry: A perspective. Souther Journal of Applied For. Vol 5, $\mathrm{N}^{\circ} 2$.

2. DUNNE, T. 1977. Evaluation of erosion conditions and trends. University of Washington In: Guidelines of watershed management. FAO Conservation Guide.

3. KUNZ, M.; AGUIRRE S.; PETERS R.; PRADO J.A. 1985. Efecto de la utilización de las plantaciones de Pinus radiata D. Don en la mantención de la productividad del sitio En: Actas primer Simposio sobre Investigación del Pinus radiata en Chile. Valdivia 1985.

4. LAL, R. Ed. 1988. Soil erosión research methods. Iowa, USA Soil and Wather Conserv. Soc

5. STONE, E.L. 1973. The impact of timber harvest on soils and water. In: Report of President Advisory Panel on Timber and the Environment. pp.427-467. U.S. Gov. Print. OfI Washington. D.C. 